\title{
SARS-CoV-2 pandemic: an opportunity for Indian traditional medicines (AYUSH)
}

\begin{abstract}
An unknown etiology of viral pneumonia was first appeared in Wuhan, China, and identified as a new corona virus $(\mathrm{nCoV})$ and known as severe acute respiratory syndrome corona virus 2 (SARS-CoV-2) and had spread worldwide. The symptoms are fever, malaise, cough, and loss of taste or smell which resolve in a few days in most cases; but may progress to respiratory distress and organ failure. Human-to-human transmission of SARS-CoV-2 was found to occur through respiratory droplets or fomites when individuals are in close contact with an infected person. Despite this, other modes such as airborne transmission and infected utensil- touch transmission are also speculated. The lack of specific treatment or vaccines makes SARS-CoV-2 more dangerous which may lead to a massive mortality rate worldwide. Currently, at present, there is no specific treatment or cure available in the western medicine system. Thus, the scientific community must think about Complementary and alternative medicinal systems available globally. Indian medicinal system includes Ayurveda, Unani, Siddha, and Homeopathy which are referred to as AYUSH. Of these, Ayurveda and Homeopathy are ancient medicinal systems accepted world-wide, which employ natural drugs of plant, animal, and mineral origin for treatment. Anecdotal data suggest that these systems have enough potential and possibilities to be employed both for the prevention and treatment of COVID-19. At present pandemic, AYUSH recommended the Homeopathy and Ayurveda as immune-boosters to combat the SARS-CoV-2. The current scenario of SARS-CoV-2 provides a golden opportunity to employ the alternative and traditional medicinal system to prevent the COVID-19 in the shadow of western science.
\end{abstract}

Keywords: COVID-19, SARS-CoV-2, AYUSH, Ayurveda, homeopathy, herbal tea
Volume 13 Issue 3 - 2020

\author{
Suneel Prajapati,' Narasimha Kumar GV'2 \\ 'Department of Microbiology, Central Research Institute for \\ Homeopathy, India \\ ${ }^{2}$ Department of Pharmacology, Anjali Chatterjee Regional \\ Research Institute for Homeopathy, India
}

\begin{abstract}
Correspondence: Suneel Prajapati, MS.c, Drug Standardization Unit, Department of Microbiology \& Molecular biology, Dr. DP Rastogi Central Research Institute for Homeopathy,A-I/I Sector 24, Noida, Uttar Pradesh, India, Tel +919873976738, Email saimpj@gmail.com
\end{abstract}

Received: May 04, 2020 | Published: May 27, 2020
Abbreviations: SARS-CoV-2, syndrome coronavirus 2; WHO, world health organization; HCQ, Hydroxy-chloroquine; TCM, traditional chinese medicine

\section{Short commentary}

Severe acute respiratory syndrome coronavirus 2 (SARS-CoV-2), the etiological agent of the (Corona Virus Disease 2019) COVID-19, is first reported in Wuhan province of China in December 2019 and now emerged as a disease of the century with high transmission rate. ${ }^{1}$ World health organization (WHO) declared this disease as a public health emergency pandemic. ${ }^{2}$ As the COVID-19 pandemic continues to spread across the world, it infected 2,995,758 and killed 204,987 peoples as of April 29, 2020. ${ }^{3}$ Due to human contact and aerosol transmission, this deadly disease has enclasped the entire globe, bringing the world economies to their knees. Doctors, scientists, and governments are searching for safe and effective treatments to help those who are sick. However, yet there is no specific treatment and cure available. At present, the SARS-CoV-2 treatment can alleviate the symptoms, such as difficulty in breathing, chest discomfort, fever, body yaks, etc. Drugs available may not directly act on viruses due to non-specificity but providing relief to infected people by reducing the complications. The scientific idea behind treating the symptoms is to help prolong a patient's life and buy time for their immune systems to kick in and recover from viral infection. Due to this, old age people, children, and immunocompromised patients may at higher risk, although a young person is not invincible from the COVID-19. Person with diabetes, cardiac problems, kidney disease, and COVID-19 may need special attention and clinical care. ${ }^{4}$ Recently an antimalarial and antiviral drug i.e. Hydroxy-chloroquine, (HCQ), Remdesivir have been directly tried on patients to combat with COVID-19 and some improvements were observed, but large trials are needed to validate their efficacy. Globally, researchers and medical fraternity are uniting to bring some promising drugs or vaccine which can save humanity. However, such invention may take time due to large clinical trials on patients with COVID-19, and this is the only way that will be able to reveal precisely whether these interventions are safe and effective. These kinds of large trials require time, money, and patience to carry out, nevertheless, they are ongoing and some of them can be effective in the future. Despite that welcome development, it may take a while before vaccines and other treatments for COVID-19 reach patients who desperately need them.

Subsequently, the scientific community must think about the Complementary and alternative systems available globally. CAM's are currently part of the medicinal system and national drug program in different countries, like in India, Srilanka, Bangladesh, China, Singapore, etc. In India, it is referred to as Indian traditional medicines and are combined under one roof termed AYUSH and is integrated with the national medicinal system in treating several diseases. AYUSH system consists of five different holistic treatment approaches that include, Ayurveda, Yoga, Unani, Sidha, and Homeopathy. Among all Ayurveda, Yoga and Homeopathy are worldwide accepted and referred to as the ancient medicinal system. Drawing on the original Ayurveda and Homeopathy classics, contemporary scientific studies, and experiential knowledge on similar clinical settings, the holistic 
approach of Indian traditional medicine focuses on prevention through lifestyle modification, dietary management, prophylactic interventions for improving the immunity and simple remedies based on presentations of the symptoms. Even so, the fact that no system of medicines has any evidence-based medicine treatment against the COVID-19 so far. Clinical interventions are being done globally. A similar strategy is initiated by the Indian Government by implementing the Ayurveda and Homeopathy system of medicine as an immune booster in improving the respiratory health of citizens.

The Indian traditional medicines emphasize on avoidance of causative factors and enhancing the self-immunity against a host of infections and this is the key characteristics feature of the classical Ayurveda and homeopathy remedy. The Indian traditional medicines interventions are well known, and historically become even more relevant by the fact that there is an elaborate description of causation and management of epidemic such as Janapadodhwamsa in Ayurveda. And Homeopathy also has been used and proven effective during the several epidemics such as Cholera, Spanish Influenza, Yellow fever, Scarlet fever, Diphtheria, Typhoid etc. ${ }^{5,6}$ Apart from these homeopathic interventions are well known for the management of few genus epidemics i.e. Japanese encephalitis, Dengue Virus and Chikungunya. ${ }^{7-9}$ Incoherence with the success of Indian traditional medicine in managing several epidemics and based on the potential of AYUSH systems in addressing the challenge and restoring health, medicines from Ayurveda like Chyavanprash, Herbal tea, and turmeric milk were recommended as immune boosters. Herbal tea was made from Basil, Cinnamon, Black pepper, Dry Ginger and Raisin) Basil leaves are rich in phytonutrients chlorophyll, vitamins, and minerals, as well as eugenol, a bioactive compound and having a various medicinal application. Holy basil is very beneficial in respiratory complications such as allergic bronchitis, cough, asthma, and eosinophilic lung disease it has a unique property to liquefy the phlegm. ${ }^{10,11}$ Cinnamon is the major source of cinnamaldehyde and trans-cinnamaldehyde (Cin), which have various biological activities apart from this its bark contains procyanidins and catechins and they possess tremendous antioxidant capacity. ${ }^{12,13}$ Black peppers can activate the circulatory system and traditionally used as a cold and flu treatment. Ginger contains chemicals called sesquiterpenes that target cold viruses. This immune-boosting spice also has antibacterial properties that can help prevent nausea similarly raisins are packed with energy and are rich in fiber, vitamins, and minerals, due to these it has also tremendous medicinal applications. Thus these traditional spices were recommended as herbal tea to boost immunity. Among homeopathic medicines, Arsenicum albums $30 \mathrm{CH}$, daily once in an empty stomach for three days as immune boosters in improving respiratory health was also recommended ${ }^{14}$. However, the above-mentioned drugs not yet clinically proved against the SARSCoV-2 infection although various studies are ongoing. Apart from AYUSH, it is noteworthy that, traditional Chinese herbal medicine (TCM) played a very crucial role in the management of COVID-19 and is part of the national health program of China. TCM played a key role in mitigating the infection it is used as palliative therapy in combination with western medicine to treat the SARS-like illness. ${ }^{15}$ TCM is now officially included in the Chinese Guideline on diagnosis and treatment of COVID-19and cured some patients in Beijing, China after applying TCM with symptomatic therapy. ${ }^{16,17}$ Given the success of TCM in managing a SARS-CoV-2 pandemic in China, it essential to explore how Ayurveda and Homeopathy work and can help in control the COVID-19 infection in India. The Indian traditional medicine especially, Ayurveda and Homeopathy have enough potential and possibilities to be employed in the management of COVID-19. Anecdotal data and historical background suggest that this system of medicines may be providing an alternative solution if studied scientifically. However, the followers of western medicine always criticize and challenge these medicinal systems from time to time. Despite this, about $70-80 \%$ of the world's population relies on alternative and traditional medicines. Therefore more research studies are required in the future to explore the scientific data to validate these drugs in a modern scientific way to prove there safety and efficacy. Although due to scanty scientific evidence this system of medicines gets very limited attention to the scientific community. In recent, it is noticed that these systems of medicine specially AYUSH, in India and TCM in China getting the attention of the scientific community and government. Such support will be helpful in the scientific advancement of these forgotten systems of medicine in the $21^{\text {st }}$ century. The current pandemic of SARS-CoV-2 also provides a golden opportunity to the alternative and traditional medicinal system to prove its importance and utility in the shadow of western science.

\section{Acknowledgments}

None.

\section{Conflicts of interest}

The authors declare that there are no conflicts of interest.

\section{Funding}

None.

\section{References}

1. Prajapati S, Sharma M, Kumar A, et al. An update on novel COVID-19 pandemic: a battle between humans and virus. Eur Rev Med Pharmacol Sci. 2020;24:819-5829.

2. World Health Organization (WHO). WHO Director-General's opening remarks at the media briefing on COVID-19 - 11 March 2020. Geneva, Switzerland: World Health Organization; 2020.

3. World Health Organization (WHO). WHO Health Emergency DashboardWHO (COVID-19). Geneva, Switzerland: World Health Organization; 2020

4. Gupta R, Ghosh A, Singh AK, et al. Clinical considerations for patients with diabetes in times of COVID-19 epidemic. Diabetes Metab Syndrome Clin Res Rev. 2020;14:3:211-212.

5. Jyotirmoy S, Rekha SD. Concept of Epidemic Diseases in Ayurveda. IJHRMLP. 2016;2:24.

6. https://homeopathyplus.com/what-arehomeopathic-prophylactics

7. Rejikumar R, Dinesh RS. A Study on the Prophylactic Efficacy of Homoeopathic Preventive Medicine against Chikungunya Fever.

8. Janardanan Nair KR, Gopinadhan S, Sreedhara Kurup TN, et al. Homoeopathic Genus Epidemicus 'Bryonia alba' as a prophylactic during an outbreak of Chikungunya in India: A cluster -randomised, double blind, placebo- controlled trial. Indian J Res Homoeopathy. 2014;8:160 165 .

9. Wadhwani GG. Homeopathic drug therapy Homeopathy in Chikungunya Fever and Post-Chikungunya Chronic Arthritis: an observational study. Homeopathy. 2013;102:93-198.

10. Liu X, Zhang M, He L, et al. Chinese herbs combined with Western medicine for severe acute respiratory syndrome (SARS). Cochrane Database Syst Rev. 2012;10:CD004882

11. http://apps.who.int/medicinedocs/en/d/Js4927e/21.html

12. Nonaka GI, Morimoto S, Nishioka I. Tannins and related compounds. Part 13. Isolation and structures of trimeric, tetrameric, and pentameric proanthicyanidins from cinnamon. Journal of the Chemical Society, Perkin Transactions 1. 1983:2139-2145. 
13. Maatta-Riihinen KR, Kähkönen MP, Törrönen AR, et al. Catechins and procyanidins in berries of vaccinium species and their antioxidant activity. J Agric Food Chem. 2005;53(22):8485-84891.

14. Ayurveda's immunity boosting measures for self-care during COVID 19 crisis. India: Ministry of AYUSH, Government of India, 2020.

15. Liu X, Zhang M, He L, et al. Chinese herbs combined with Western medicine for severe acute respiratory syndrome (SARS). Cochrane Database Syst Rev. 2012;10:CD004882.
16. A patient diagnosed with 2019-nCoV pneumonia was discharged from Beijing.

17. National health commission of the people's republic of china. Guideline on diagnosis and treatment of covid-19 (trial 6th edition). 